\title{
A sensorless control for a variable speed wind turbine operating at partial load
}

\author{
Ronilson Rocha \\ School of Mines, Department of Engineering of Control and Automation, Federal University of Ouro Preto, Campus Morro do Cruzeiro, $35400-000$ Ouro Preto, MG, Brazil
}

\section{A R T I C L E I N F O}

\section{Article history:}

Received 3 August 2009

Accepted 4 June 2010

Available online 29 June 2010

\section{Keywords:}

Wind energy

Stall regulated wind turbine

Sensorless control

LQG/LTR methodology

\begin{abstract}
A B S T R A C T
This paper presents a sensorless control for a variable speed wind turbine (WT) operating at partial load in order to eliminate the direct measurement of the wind speed. In this proposal, the estimated aerodynamic torque is used to determine the optimal reference of the speed control for maximum energy conversion. The maximization of the efficiency on energy conversion and the minimization of detrimental dynamical loads are control trade-offs considered in the design of an optimal discrete-time feedback LQG/LTR controller for the Wind Energy Conversion System (WECS), which is based on the optimization of a quadratic cost function. The performance of the proposed control when the WT is submitted to a gust or step variation on wind speed is evaluated from computational simulations. It is also presented some proposals for sensorless control of the electrical generator.
\end{abstract}

(c) 2010 Elsevier Ltd. All rights reserved.

\section{Introduction}

Due to significant cost reductions, improved efficiency and the increased useful life provided by the technological development of wind energy conversion systems (WECS), wind power is considered one of the most attractive solutions to energy problems. The basic configuration of a WECS is shown in Fig. 1. It is composed by a wind turbine (WT) coupled to an electrical generator, directly or through gear-box. In spite of its simplicity, the WECS represents an interesting problem in viewpoint of the control theory, since it is a nonlinear system, subjected to cyclical disturbances caused by operational phenomena, and highly dependent of a stochastic variable characterized by sudden variations. Thus, the quality of a WECS controller is determined by its capacity to deal with model uncertainties, exogenous stochastic signals, and periodic disturbances.

Although classical methods are traditionally utilized in WECS control, these solutions are not completely adequate since the resulting controllers do not provide sufficient damping to system and the necessary robustness to uncertainties, such as parameter variations, nonlinear behavior, etc [ $1-5]$. In this context, the linear quadratic gaussian controller with loop transfer recovery (LQG/LTR) consists in an interesting alternative for WECS, since it combines the necessary characteristics to assure the stability margin, disturbance attenuation and a reasonable robustness to model uncertainties [6,7]. Furthermore, the control trade-offs for a WECS can be easily translated into a quadratic cost function [3,8], which is inherent to design of an optimal linear feedback LQG/LTR controller.

\footnotetext{
E-mail address: rocha@em.ufop.br.
}

The information related to wind speed is essential for the control of a WECS. However, the use of anemometers to measure the wind speed can represent a serious obstacle to implement a robust control structure for a WECS $[9,10]$ :

- The possible contamination of the reference signal by the wind fluctuation, which can be solved filtering the measurement signal;

- The wind speed can not be measured exactly in the WT rotor, which difficulties the exact evaluation of the available power for energy conversion;

- The installation of the anemometer can reduce the reliability of the measure of wind speed due to aerodynamic interference, shadowing effects, turbulences, etc.

Another fundamental information for WECS control is the position or the rotation of mechanical shaft, which can be measured using tachometers, encoders and resolvers. However, the use of these transducers can also introduce others problems:

- These transducers are often one of the most expensive and fragile components in a WECS, principally for small and medium power systems;

- The space limitations and difficult access to the mechanical shaft can implicate in restrictions to use of a speed sensor;

- The additional wires for electrical connections of a speed sensor can increase the vulnerability of the system to electromagnetic interferences (EMI);

- Problems related to assembling and maintenance. 


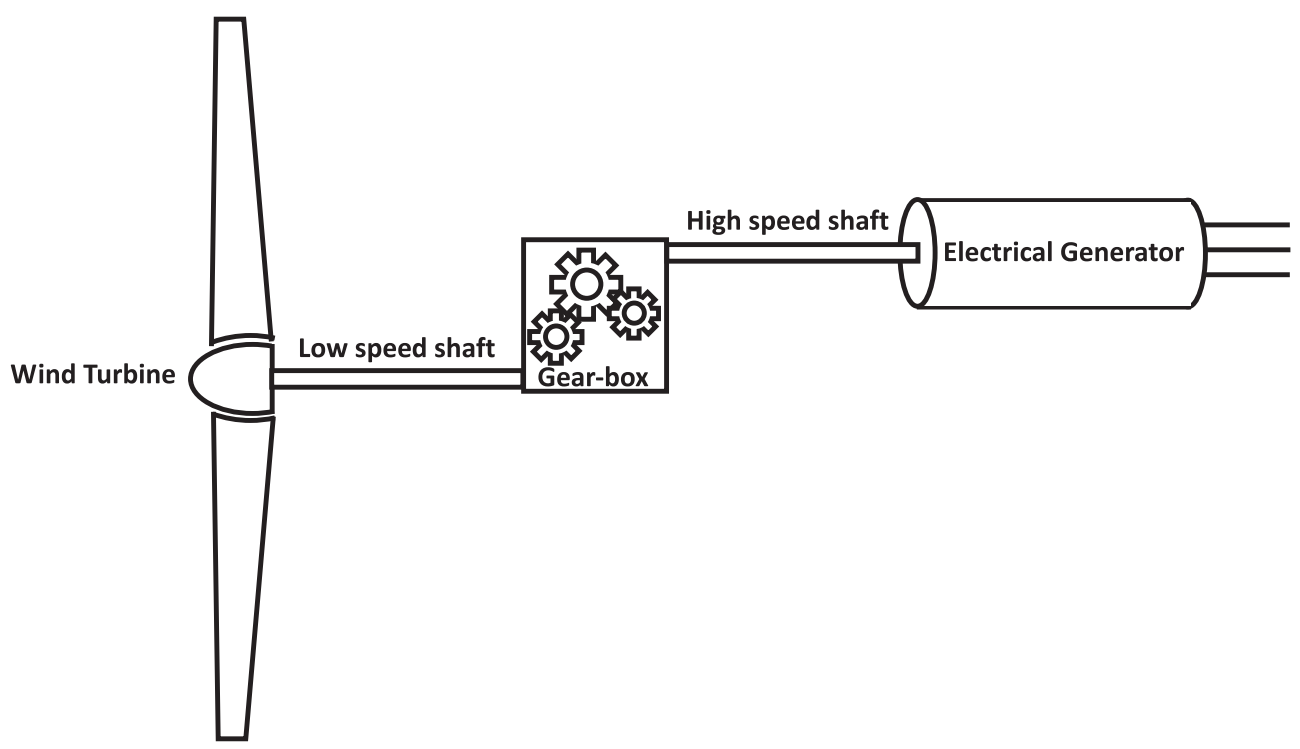

Fig. 1. Basic WECS configuration.

Thus, the direct measurement of the wind speed, position and rotation of the mechanical shaft can represent a great obstacle to the implementation of a more efficient, robust and cheap WECS, since the related sensors can be extremely expensive and introduce several uncertainties in the system. In this context, the elimination of the direct measurements of the mechanical variables in the control system consists in a very attractive perspective in the technical and economical viewpoints for WECS design, principally for small and medium systems.

This paper presents a sensorless control for a variable speed WT operating at partial load, aiming the elimination of the direct measurement of the wind speed. In this proposal, the estimated aerodynamic torque is used to determine the optimal reference of the speed control for maximum energy conversion. The maximization of the efficiency on energy conversion and the minimization of detrimental dynamical loads are control trade-offs considered in the design of an optimal discrete-time feedback LQG/LTR controller for the Wind Energy Conversion System (WECS), which is based on the optimization of a quadratic cost function. The performance of the proposed control when the WT is submitted to a gust or step variation on wind speed is evaluated from computational simulations. It is also presented some proposals for sensorless control of the electrical generator.

\section{Nonlinear WECS model}

The WECS dynamics are presented in Fig. 2. For the computational simulation of the system, it is necessary a complex and consistent nonlinear WT model that consider several operational phenomena, such as losses, wind shear, ripple torque, and turbulence. Assuming a single dimensional random process, a nonlinear dynamic model for WT system can be divided in four distinct subsystems [11]: wind, aerodynamics, driven train and electrical generator. In the approach adopted in this paper, the structural dynamics are neglected and considered as model uncertainties.

\subsection{Wind}

An adequate model of the wind is necessary to predict the dynamics of a WECS. Although the wind is a multidimensional stochastic process, which depends on the time and spatial coordinates, a two dimensional model is generally enough to describe adequately this phenomenon [11]. The wind speed $V_{H}$ at the reference height $H$ can be given by $[12,13]$ :

$V_{H}=\bar{V}+\Delta V+V_{G}+V_{R}$

where $\bar{V}$ is the effective average of the wind speed over the WT rotor and $\Delta V$ is the wind fluctuation given by:

$\Delta V=2 \sum_{i=1}^{N}\left[S_{v}\left(\omega_{i}\right) \Delta \omega\right]^{\frac{1}{2}} \cos \left(\omega_{i} t+\Phi_{i}\right)$

where $\omega_{i}=(i-(1 / 2)) \Delta \omega, \Phi_{i}$ is an independent random variable with uniform density in the interval of $0-2 \pi$ and $S_{v}\left(\omega_{i}\right)$ is the power spectral density, given by [11]:

$$
S_{v}\left(\omega_{i}\right)=\frac{2 K_{N} F^{2}\left|\omega_{i}\right|}{\pi^{2}\left[1+\left(\frac{F \omega_{i}}{\mu \pi}\right)^{2}\right]^{\frac{4}{3}}}
$$

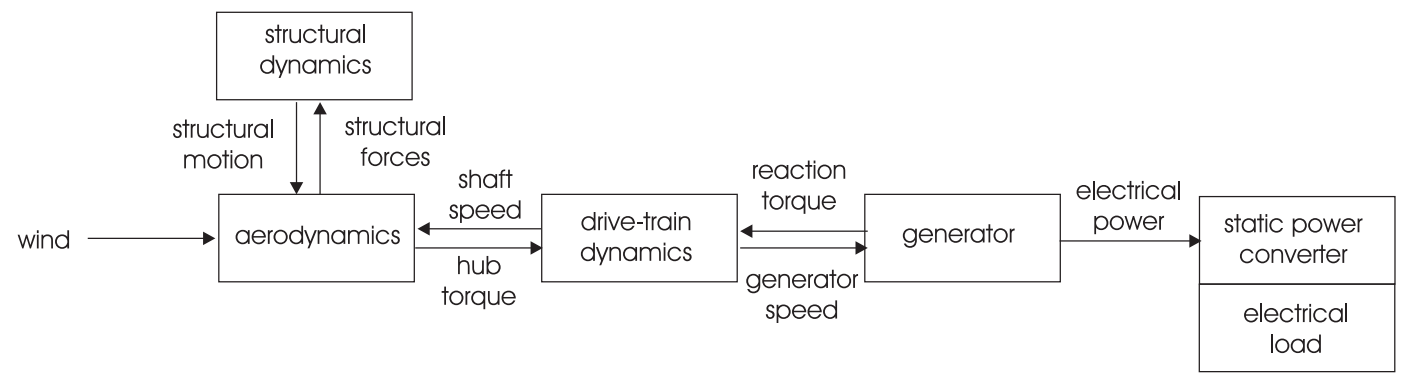

Fig. 2. WECS dynamics. 
where $K_{N}$ is the superficial drag coefficient, $F$ is the turbulence scale, and $\mu$ is the average of the wind speed in the reference height. For good results, it is suggested $N=50$ and $\Delta \omega$ between 0.5 and 2.0 $\mathrm{rad} / \mathrm{s}$ [14]. Since the wind speed depends of the height due to "wind shear", an approach to compute the wind speed at a specific height $h$ is given by $[11,15,16]$ :

$V_{h}=V_{H}\left(\frac{h}{H}\right)^{a}$

where $a$ is a coefficient that depends of the local topography. Considering the blade geometry, the wind speed $V_{i}$ in the representative point $3 / 4$ of the cord of the $i$-th blade can be expressed as:

$V_{i}=V_{H}\left\{1+\frac{3}{4} \frac{R}{H} \sin \left(\theta_{B i}\right)\right\}^{a}$

where $\theta_{B_{i}}$ is the spacial angle of the $i$-th blade. An essential component to study the dynamical behavior of a WECS is the discrete longitudinal gust $V_{G}$. Considering that a wind gust begins at instant $T_{G}$ and finishes after $\Delta T_{G}$, this component can be estimated as $[14,17,18]$ :

$V_{G}=\frac{3 \bar{V}_{H}}{2 \ln \left(\frac{H}{h_{o}}\right)}\left\{1-\exp \left(-\frac{\bar{V}_{h} \Delta T_{G}}{1.48 h}\right)\right\}^{\frac{1}{2}}\left[1-\cos \left(2 \pi \frac{t-T_{G}}{\Delta T_{G}}\right)\right]$

for $T_{G}<t<T_{G}+\Delta T_{G}$, where the parameter $h_{0}$ is the roughness height. Another important component of the wind speed is the ramp $V_{R}$ described as [14]:

$V_{R}=V_{R M A X}\left(\frac{t-T_{R}}{\Delta T_{R}}\right)$

for $T_{R}<t<T_{R}+\Delta T_{R}$, where $V_{R M A X}$ is the peek of the ramp, $T_{R}$ is the initial instant when the ramp begins and $\Delta T_{R}$ is ramp duration. For small $\Delta T_{R}$, the ramp component can be used as an approach of the wind step.

\subsection{WT aerodynamics}

An accurate model for the aerodynamic behavior of a WT is often very difficult to obtain due to the uncertainties in its description. The aerodynamic behavior of the WT is nonlinear, dependent of wind speed and may change over time due to contamination of blade surfaces. In general, the best approach to evaluate the aerodynamic torque $Q_{a}$ uses dimensionless coefficients $C_{p}$ and $C_{q}$, which respectively express the WT ability to convert kinetic energy of moving air into mechanical power or torque $[8,19]$. Besides dependency of constructive aspects of the WT blades, both coefficients $C_{p}$ and $C_{q}$ are nonlinear functions of the yaw angle $\theta$ and a parameter known as tip-speed ratio $\lambda$, which is defined as:

$\lambda=\frac{R \omega_{t}}{V}$

where $R=$ turbine radius, $\omega_{t}=\mathrm{WT}$ speed and $V=$ wind speed. Admitting that WT is always aligned with wind direction, i.e. $\theta=0^{\circ}$, the aerodynamic torque $Q_{a}$ in a blade of WT is given by $[8,11,15]$ :

$Q_{a}=\frac{1}{2} \rho A R \frac{C_{p}(\lambda)}{\lambda} V^{2}=\frac{1}{2} \rho A R C_{q}(\lambda) V^{2}$

where $\rho=$ air density and $A=$ rotor area. Typical $C_{p} \times \lambda$ and $C_{q} \times \lambda$ characteristics of a WT are shown in the Fig. 3.

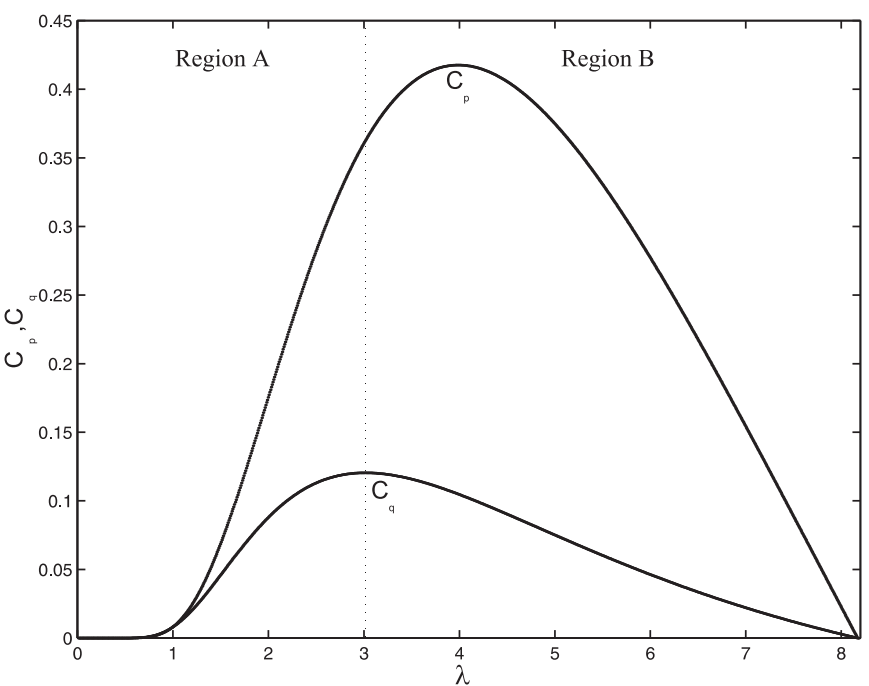

Fig. 3. Aerodynamic characteristics of a WT.

\subsection{Driven train}

The driven train can be modeled as a set of masses coupled through flexible connections as shown in Fig. $4[8,20]$. Although the gear-box has nonlinear characteristics [21], this component can be supposed ideal. Using the classical rotational dynamics to describe the coupling system of a WT with $n$ blades:

- $i$-th blade:

$J_{i} \dot{\omega}_{i}+D_{i} \omega_{i}=Q_{a i}-D_{i h}\left(\omega_{i}-\omega_{h}\right)-Q_{m i h}$

- hub:

$J_{h} \dot{\omega}_{h}+D_{h} \omega_{h}=\sum_{i=1}^{n}\left[D_{i h}\left(\omega_{i}-\omega_{h}\right)+Q_{m i h}\right]-D_{h g}\left(\omega_{h}-\omega_{g}\right)-Q_{m h g}$

- electrical generator:

$J_{g} \dot{\omega}_{g}+D_{g} \omega_{g}=D_{h g}\left(\omega_{h}-\omega_{g}\right)+Q_{m h g}-Q_{g}$

- shaft torques:

$\dot{Q}_{\operatorname{mih}}=K_{i h}\left(\omega_{i}-\omega_{h}\right)$

$\dot{Q}_{m h g}=K_{h g}\left(\omega_{h}-\omega_{g}\right)$

where $\omega_{i}=i$-th blade speed, $\omega_{h}=$ hub speed, $\omega_{g}=$ generator speed, $J_{i}=i$-th blade inertia, $J_{h}=$ hub inertia, $J_{g}=$ generator inertia (including the gear-box inertia), $D_{i}=i$-th blade damping, $D_{h}=$ hub damping, $D_{g}=$ generator damping, $D_{i h}=i$-th blade-hub connection damping, $D_{\text {hg }}=$ shaft damping, $K_{i h}=i$-th blade-hub connection stiffness, $K_{h g}=$ shaft stiffness, $Q_{a i}=i$-th aerodynamic torque, $Q_{m i h}=i$-th blade torque, $Q_{m h g}=$ shaft torque and $Q_{g}=$ generator torque.

\subsection{Electrical generator}

The electrical generator constitutes the link between the rotational mechanical energy and the available electrical power to 


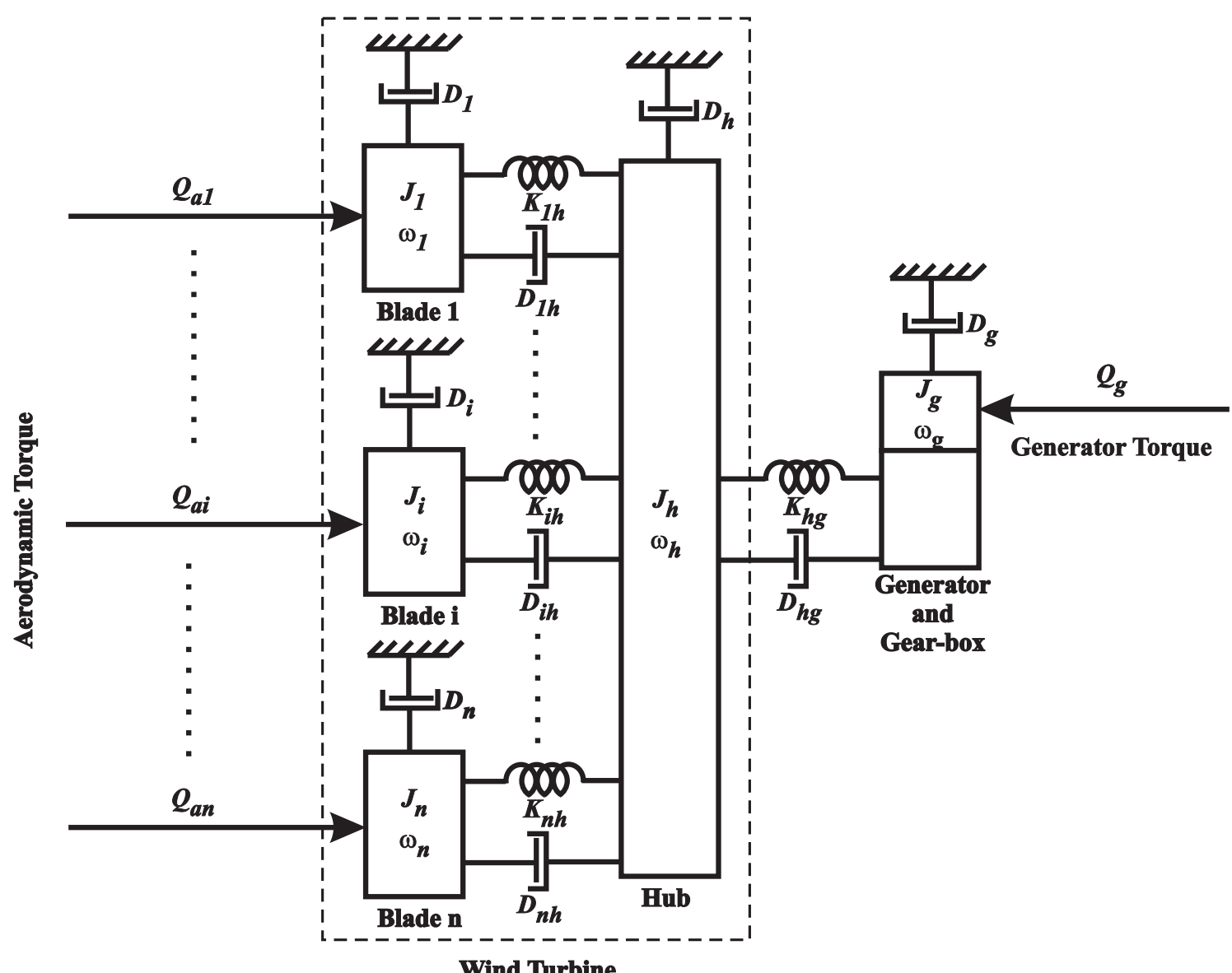

Fig. 4. Driven train model.

costumers. Since the dynamics of electrical systems are extremely fast when compared to mechanical coupling, a quasi-static model can be assumed for electrical generator. Considering the use of a current controlled static converter as the interface between the generator and the electrical load, the generator torque $Q_{g}$ is perfectly adjustable in all operating bandwidth and virtually independent from WECS dynamics [8], consisting in the only control input of a stall regulated WT.

\section{Nominal linearized model for control design}

For robust control design, it is necessary to derive a simplified and linearized mathematical model of the WT. Observing the typical characteristic $C_{q} \times \lambda$ of a WT shown in Fig. 3, two distinct operating regions can be identified $[8,22,23]$ :

- The stall region $(A)$, characterized by a positive slope of the curve $C_{q} \times \lambda$, where the operation is unstable and a sudden and significant drop in the aerodynamic torque occurs due to phenomenon known as aerodynamic "stall";

- The normal operation region (B), characterized by a negative slope of the curve $C_{q} \times \lambda$, which corresponds to normal operation of WT.

The linearization of the aerodynamic torque $Q_{a}$ can be performed in the specific point of maximum $C_{p}$, which is always situated in the normal operating region. In this operating point, $\lambda=\lambda_{\text {opt }}$ and the derivatives $\partial C_{p} / \partial \omega_{t}$ and $\partial C_{p} / \partial V$ are null. The linearized aerodynamic torque $Q_{a}$ around maximum $C_{p}$ is given by:
$\dot{Q}_{a}=\alpha \dot{V}+\gamma \dot{\omega}_{t}$

where $\alpha$ is the scaling factor of the torque disturbance due to wind variations $\dot{V}$, and $\gamma$ denotes the acceleration feedback coefficient from drive-train. In steady state, $\dot{V}$ is the wind fluctuation, which can be assumed as an uncorrelated in the time zero-mean Gaussian stochastic signal [11]. Considering $\bar{V}$ as the nominal wind speed, the coefficients $\alpha$ and $\gamma$ can be computed from WT data as:

$\alpha=\left.\frac{\partial Q_{a}}{\partial V}\right|_{\lambda_{\text {opt }}}=\frac{3}{2} \rho A R \frac{C_{p \max }}{\lambda_{\text {opt }}} \bar{V}$

$\gamma=\left.\frac{\partial Q_{a}}{\partial \omega_{t}}\right|_{\lambda_{o p t}}=-\frac{1}{2} \rho A R^{2} \frac{C_{p \max }}{\lambda_{o p t}^{2}} \bar{V}$

Although a real mechanical drive train has rigid disks connected by a flexible shaft, where the inertias and compliances are distributed along its length, an approximated lumped 2-mass model presented in Fig. 5 is enough to obtain a good indication of the dynamical loads for control design [8,15]. Admitting an ideal gearbox and reducing all quantities to primary side, the mechanical coupling can be described using classical rotational dynamics [12]:

$$
\begin{aligned}
& J_{t} \dot{\omega}_{t}+D_{t} \omega_{t}=Q_{a}-Q_{m h g}-D_{h g}\left(\omega_{t}-\omega_{g}\right) \\
& J_{g} \dot{\omega}_{g}+D_{g} \omega_{g}=D_{h g}\left(\omega_{t}-\omega_{g}\right)+Q_{m h g}-Q_{g} \\
& \dot{Q}_{m h g}=K_{h g}\left(\omega_{t}-\omega_{g}\right)
\end{aligned}
$$




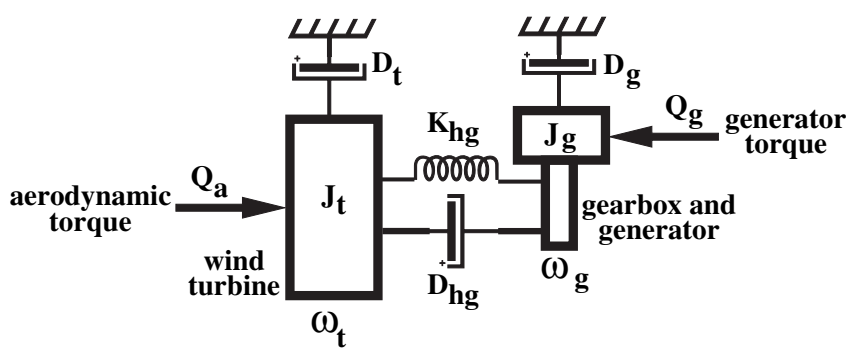

Fig. 5. Approximated lumped drive train model.

where $\omega_{t}=\mathrm{WT}$ speed, $J_{t}=$ total WT inertia and $D_{t}=$ total WT damping.

The structural dynamics and the nonlinearities can be admitted as uncertainties. Thus, the nominal linearized state model of a stall regulated WT for control design is given by:

$\dot{\boldsymbol{x}}(t)=\mathbf{A x}(t)+\mathbf{b} Q_{g}(t)+\psi$

$y(t)=\mathbf{c x}(t)+\xi$

where $\boldsymbol{x}(t)=\left[\begin{array}{llll}Q_{a} & \omega_{t} & \omega_{g} & Q_{m}\end{array}\right]^{\prime}$ and the disturbance input on the system due to wind fluctuation $\dot{V}$ is given by $\psi=\left[\begin{array}{lll}\alpha \dot{V} & 0 & 0\end{array}\right]^{\prime}$. Due to practical constraint relatives to assembling, cost and maintenance of sensors, the generator rotation $\omega_{g}$, contaminated by the measurement noise $\xi$, is considered the only measured output $y$. The matrices $\mathbf{A}, \mathbf{b}$ and $\mathbf{c}$ are given by:

$\mathbf{A}=\left[\begin{array}{cccc}\frac{\gamma}{J_{t}} & -\frac{\gamma\left(D_{t}+D_{h g}\right)}{J_{t}} & \frac{\gamma D_{h g}}{J_{t}} & -\frac{\gamma}{J_{t}} \\ \frac{1}{J_{t}} & -\frac{D_{t}+D_{h g}}{J_{t}} & \frac{D_{h g}}{J_{t}} & -\frac{1}{J_{t}} \\ 0 & \frac{D_{h g}}{J_{g}} & -\frac{D_{h g} D_{g}}{J_{g}} & \frac{1}{J_{g}} \\ 0 & K_{h g} & -K_{h g} & 0\end{array}\right]$

$\mathbf{b}=\left[\begin{array}{llll}0 & 0 & -\frac{1}{J_{g}} & 0\end{array}\right]^{\prime}$

$\mathbf{c}=\left[\begin{array}{llll}0 & 0 & 1 & 0\end{array}\right]$

\section{Some control requirements}

For medium- to large-scale systems, the controller design can be difficult from a numerical point of view, since the involved matrix operations tend to be ill-conditioned [4]. Although both speed and power can be selected as the controlled variable in a WECS, the speed control is considered dynamically better than the power control [24]. In this case, the WECS must be operated at fixed $\lambda_{\text {opt }}$ to maximize the energy conversion. This strategy can compromise the control performance due to the direct dependence of the rotation reference with the wind speed, which is always varying and can not be measured exactly at the WT rotor.

Another problem in a WECS is the propagation of vibrations and ripple torque on the system with undesirable consequences on energy quality and lifetime of several system components. Sudden variations in the wind can excite torsional modes, which can cause unacceptable mechanical efforts on the drive train. Although the aerodynamic stall provides the speed limitation for high wind speed, it is necessary to avoid operation in the stall region in order to obtain a better WECS performance [15]. Thus, the control design has to establish a trade-off between the dynamical loads in the system and the increase of the energy conversion. Given the multiple aspects of WECS global efficiency (reliability, availability, remote operation, quality of captured power, etc.), the WECS optimal control implies the necessity of adopting mixed-criteria optimization indices [25].

The poles of the closed-loop system must be assigned as far away as possible from the frequencies related to the greatest energy density of dynamical loads. Furthermore, it is necessary to obtain sufficient gain and phase margins to assure a safe stability margin, since the WECS uncertainties can not be quantified due to the complexity of interactions between WT and wind field. According to Leith e Leithead [12], practical experience suggests that a gain margin of $10 \mathrm{~dB}$ and a phase margin of roughly $60^{\circ}$ are sufficient to assure the control design robustness for any WECS.

\section{LQG/LTR methodology}

The discrete-time LQG/LTR methodology consists in the formal establishment of a quadratic performance index to synthesize a linear feedback regulator $\mathbf{K}_{\mathbf{c}}$. Considering a discrete-time state model $(\boldsymbol{\Phi}, \boldsymbol{\Gamma}, \boldsymbol{\Psi}, \boldsymbol{\Pi})$, the Linear Quadratic Regulator (LQR) provides a control signal that minimizes the following cost function $[7,26]$ :

$J=\sum_{k=0}^{\infty}\left[\boldsymbol{z}^{\prime}(k) \mathbf{Q z}(k)+\boldsymbol{u}^{\prime}(k) \mathbf{R u}(k)\right]$

where $\mathbf{z}(k)$ corresponds to target outputs. The targets and the control input are weighted respectively by matrices $\mathbf{Q}$ and $\mathbf{R}$, and the resulting discrete-time regulator $\mathbf{K}_{\mathbf{c}}$ is given by:

$\mathbf{K}_{\mathbf{c}}=\left[\mathbf{R}+\Gamma^{\prime} \mathbf{P} \Gamma\right]^{-1} \Gamma^{\prime} \mathbf{P} \Phi$

where $\mathbf{P}$ is the definite positive solution of the following discretetime Riccati equation:

$\mathbf{P}=\mathbf{Q}+\boldsymbol{\Phi}^{\prime} \mathbf{P} \boldsymbol{\Phi}-\boldsymbol{\Phi}^{\prime} \mathbf{P} \boldsymbol{\Gamma}\left[\mathbf{R}+\Gamma^{\prime} \mathbf{P} \Gamma\right]^{-1} \Gamma^{\prime} \mathbf{P} \boldsymbol{\Phi}$

Since the LQR provides a infinite gain margin and at least $60^{\circ}$ for phase margin [7], which is enough to assure the WECS stability, the regulator design can be concentrated in to obtain an effective performance for dynamical response. An adequate choice of targets $\mathbf{z}$ and weighting matrices $\mathbf{Q}$ and $\mathbf{R}$ allows to establish a quadratic performance index of the LQR similar to the cost function for WECS control described by Leithead et al. [3], which involves the trade-offs between efficiency on energy conversion and the dynamical loads.

The optimization of the energy conversion is an important control requirement for variable speed WECS, and depends on the accuracy of speed control. Thus, the speed error $\varepsilon=\omega_{\text {ref }}-\omega_{g}$ can be used as an indication of efficiency conversion, and its inclusion in the LQR performance index implies a model expansion. In this way, the state model given by eqs. (21) and (22) is discretized for $\left(\mathbf{A}_{\mathbf{d}}, \mathbf{B}_{\mathbf{d}}, \mathbf{C}_{\mathbf{d}}, \mathbf{D}_{\mathbf{d}}\right)$ and augmented to insert the speed error $\varepsilon$ as a new state variable. The following discrete-time state model is then obtained:

$\overline{\boldsymbol{x}}(k+1)=\overline{\boldsymbol{\Phi}} \overline{\mathbf{x}}(k)+\overline{\boldsymbol{\Gamma}} Q_{g}(k)$

$\overline{\mathbf{y}}(k)=\overline{\mathbf{\Psi}} \overline{\mathbf{x}}(k)$

where $\varepsilon(k)$ is the discrete summation of the speed error, the new state vector is $\overline{\mathbf{x}}(k)=\left[\begin{array}{ll}\varepsilon(k) & \mathbf{x}^{\prime}(k)\end{array}\right]^{\prime}$ and $\overline{\mathbf{y}}(k)$ corresponds to new output. For discrete-time LQG/LTR controller design, the inclusion of this integral action assures the elimination of steady state errors. Hence the discrete-time matrices $\overline{\boldsymbol{\Phi}}, \overline{\boldsymbol{\Gamma}}$ and $\overline{\boldsymbol{\Psi}}$ are given by:

$\overline{\boldsymbol{\Phi}}=\left[\begin{array}{cc}1 & -\mathbf{C}_{\mathbf{d}} \mathbf{A}_{\mathbf{d}} \\ 0_{4 \times 1} & \mathbf{A}_{\mathbf{d}}\end{array}\right]$ 
$\overline{\boldsymbol{\Gamma}}=\left[\begin{array}{ll}-\mathbf{C}_{\mathbf{d}} \mathbf{B}_{\mathbf{d}} & \mathbf{B}_{\mathbf{d}}\end{array}\right]^{\prime}$

$\overline{\mathbf{\Psi}}=\left[\begin{array}{ll}1 & 0_{1 \times 4}\end{array}\right]$

Another important control requirement is to minimization of the detrimental dynamical loads, which are basically determined by mechanical torques and speeds. One of these dynamical loads are the torsional modes, which depend on the difference $\Delta \omega=\omega_{t}-\omega_{g}$. To reduce the stress on the shaft, the variations on the shaft torque $Q_{s}$ must be minimized over all bandwidth. The control design has also to minimize the effects of wind fluctuation and ripple torque over the energy delivered to electrical load, which is represented in the WECS model by the generator torque $Q_{g}$. Thus, the targets can be chosen as $\mathbf{z}(k)=\mathbf{M} \overline{\mathbf{x}}(k)$, where the matrix $\mathbf{M}$ is:

$\mathbf{M}=\left[\begin{array}{ccccc}1 & 0 & 0 & 0 & 0 \\ 0 & 0 & 1 & -1 & 0 \\ 0 & 0 & 1 & 0 & 0 \\ 0 & 0 & 0 & 1 & 0 \\ 0 & 0 & D_{h g} & -D_{h g} & 1\end{array}\right]$

Since the weighting matrices are selected as $\mathbf{Q}=\operatorname{diag}\left(w_{1}, w_{2}, w_{3}\right.$, $\left.w_{4}, w_{5}\right)$ and $\mathbf{R}=w_{6}$, the performance index of the LQR regulator is converted into:

$$
\begin{aligned}
J= & \sum_{0}^{\infty}\left[w_{1}(\varepsilon)^{2}+w_{2}(\Delta \omega)^{2}+w_{3}\left(\omega_{t}\right)^{2}+w_{4}\left(\omega_{g}\right)^{2}\right. \\
& \left.+w_{5}\left(Q_{s}\right)^{2}+w_{6}\left(Q_{g}\right)^{2}\right]
\end{aligned}
$$

The relative importance of each target on the index to be minimized by the LQR action is determined by its respective weight $w_{i}$, which establishes the intensity of the control action on a specified target. The use of weights dependent on frequency (weighting functions) increases the flexibility on targets manipulation, allowing to penalize selectively a determined bandwidth. However, weighting functions increases the controller complexity due to addition of extra dynamics to the original model. The selection of the weights $w_{i}$ depends strongly on the system configuration and the inevitable design trade-offs.

The complement of the LQG/LTR controller design consists in the determination of the discrete-time Kalman filter for optimal estimation of the state variables, which will be used as the exact measurements by LQR. This filter estimates the state vector $\widehat{\mathbf{x}}(k / k)$ from actual measurement vector $\mathbf{y}(k)$, taking the following form [27]:

$$
\begin{aligned}
& \widehat{\mathbf{x}}(k+1 / k)=\overline{\boldsymbol{\Phi}} \widehat{\mathbf{x}}(k / k-1)+\overline{\boldsymbol{\Gamma}} \boldsymbol{u}(k)-\overline{\boldsymbol{\Phi}} \mathbf{K}_{\mathbf{f}}(\overline{\boldsymbol{\Psi}} \widehat{\mathbf{x}}(k / k-1)-\boldsymbol{y}(k)) \\
& \widehat{\mathbf{x}}(k / k)=\widehat{\mathbf{x}}(k / k-1)-\mathbf{K}_{\mathbf{f}}(\overline{\boldsymbol{\Psi}} \widehat{\mathbf{x}}(k / k-1)-\boldsymbol{y}(k))
\end{aligned}
$$

Assuming the state and measurement noise covariance matrices $\mathbf{W}$ and $\mathbf{V}, \mathbf{K}_{\mathbf{f}}$ is given by:

$\mathbf{K}_{\mathbf{f}}=\mathbf{P} \overline{\boldsymbol{\Psi}}^{\prime}\left(\overline{\boldsymbol{\Psi}} \mathbf{P} \overline{\boldsymbol{\Psi}}^{\prime}+\mathbf{V}\right)^{-1}$

where $\mathbf{P}$ is the positive semidefinite solution of the Riccati equation:

$\mathbf{P}=\overline{\boldsymbol{\Phi}} \mathbf{P} \overline{\boldsymbol{\Phi}}^{\prime}-\overline{\boldsymbol{\Phi}} \mathbf{P} \overline{\boldsymbol{\Psi}}^{\prime}\left(\overline{\boldsymbol{\Psi}} \mathbf{P} \overline{\boldsymbol{\Psi}}^{\prime}+\boldsymbol{V}\right)^{-1} \overline{\boldsymbol{\Psi}} \mathbf{P} \overline{\boldsymbol{\Phi}}^{\prime}+\mathbf{W}$

The complete WECS controller is synthesized by connection of the Kalman filter to LQR, as shown in Fig. 6. However, the inclusion of the Kalman filter can imply in the degradation of robustness and performance properties obtained in the LQR design. It is possible to recover these properties through the LTR procedure (Loop Transfer Recovery), which consists in an adequate choice of the covariance matrices $\mathbf{V}$ and $\mathbf{W}$ in the Kalman filter design $[6,7,27]$. This procedure also allows the asymptotic closed-loop poles placement, avoiding undesirable frequencies [26].

\section{Sensorless control}

For maximum energy conversion, the speed reference $\omega_{\text {ref }}$ must be directly proportional to wind speed to assure the WT operation at $\lambda_{\text {opt }}$. This strategy can reduce the control robustness due to problems related to wind speed measurement. However, the optimal speed reference $\omega_{\text {ref }}$ for the control system can be indirectly obtained, avoiding the necessity to measure the wind speed. From eq. (9), the following relationship is obtained considering the WECS operating at $\lambda_{\text {opt }}$ :

$\omega_{\text {ref }}=K_{q a} \sqrt{Q_{a}}$

where $K_{q a}$ is a constant value given by:

$K_{q a}=\frac{\lambda_{o p t}}{R} \sqrt{\frac{2 \lambda_{o p t}}{\rho A R C_{p \max }}}$

Since the aerodynamic torque $Q_{a}$ measurement is not directly available, it is estimated from the preceding data using a Kalman predictor, as shown the Fig. 7. Considering the nominal WECS model described in section 2, the dynamics of the Kalman predictor is given by eq. (29), where the gain $\mathbf{K}_{\mathbf{f}}$ is computed using the eqs. (31) and (32) [27]. The covariance matrices $\mathbf{W}$ and $\mathbf{V}$ must be selected for a slowest response if compared to feedback controller, aiming a good filtering of the speed reference and the decoupling between predictor and controller dynamics.

There are several proposed strategies for the elimination of a sensor to measure the shaft speed of an electrical motor that can used in the case of the generator. The most of these

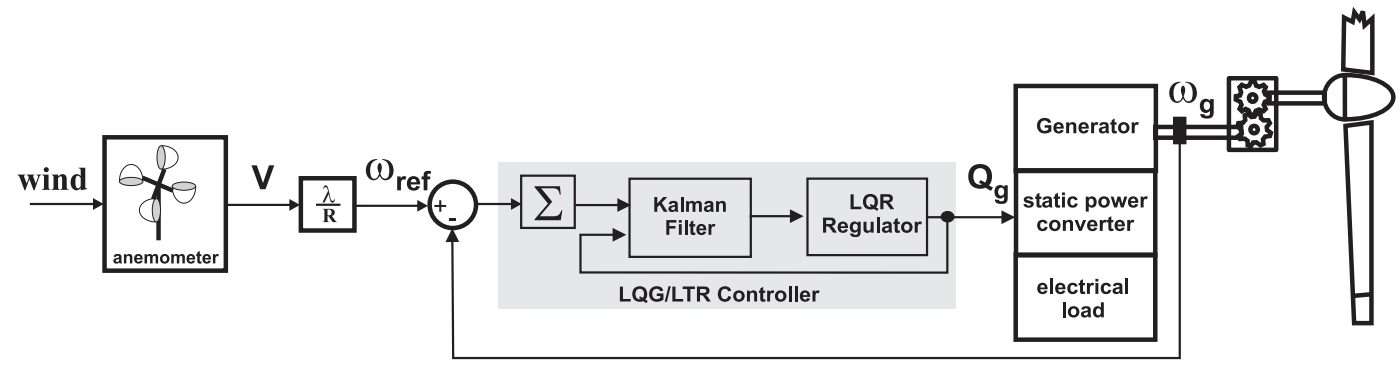

Fig. 6. LQG/LTR control structure for WECS 


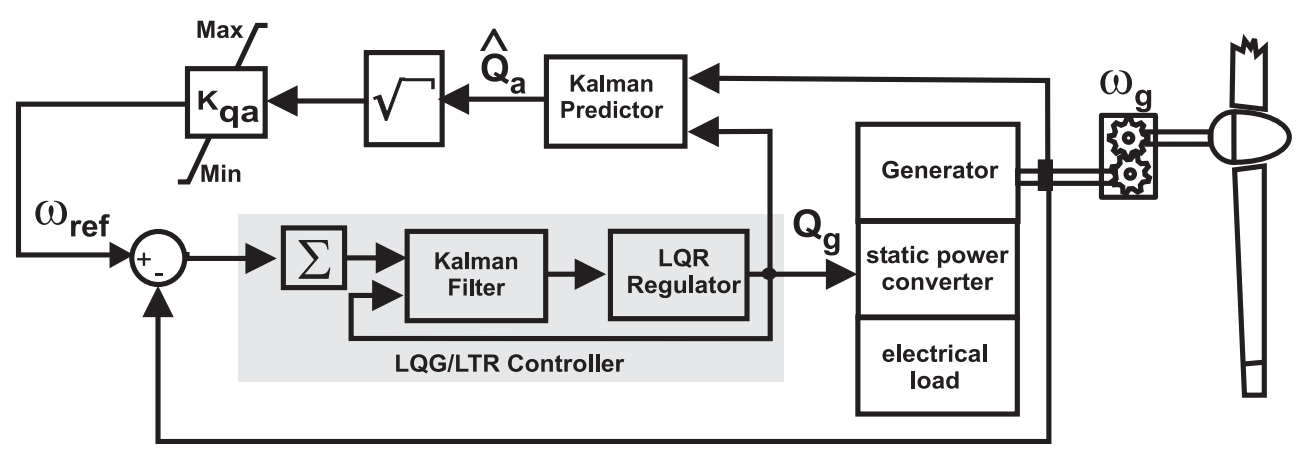

Fig. 7. Sensorless controller for WT: Optimal speed reference determination.

strategies is based in the estimative of back emf (in the case of the generator, emf), a phenomenon that depends of the rotor speed which can be estimated from the machine voltage and current measurements [28-31]: from a dynamical model, an adaptive observer is designed to estimate the states and the rotor speed. The drawback of this strategy is its inherent dependence with the machine parameters, which becomes the speed estimation sensible to parameter uncertainties [32]. The second strategy is the injection of a high frequency voltage wave in the machine terminals to induce a current signal, which depends of the rotor position [33]. However, this strategy requires constructive modifications in the machine aiming to avoid torque pulsation [32]. The third strategy consists in the monitoring of the harmonic components produced by rotor slots, which filtering provides signal equivalent to an incremental encoder which resolution is $p\left(N_{r}+1\right)$ pulses per revolution, where $p=$ number of pole pairs of the machine and $N_{r}=$ number of slots per pole pairs [34].

\section{Simulation results}

The performance of the proposed sensorless control structure is verified from computational simulations considering a Horizontal Axis WT shown in the Fig. 8 with two blades of $45.72 \mathrm{~m}$

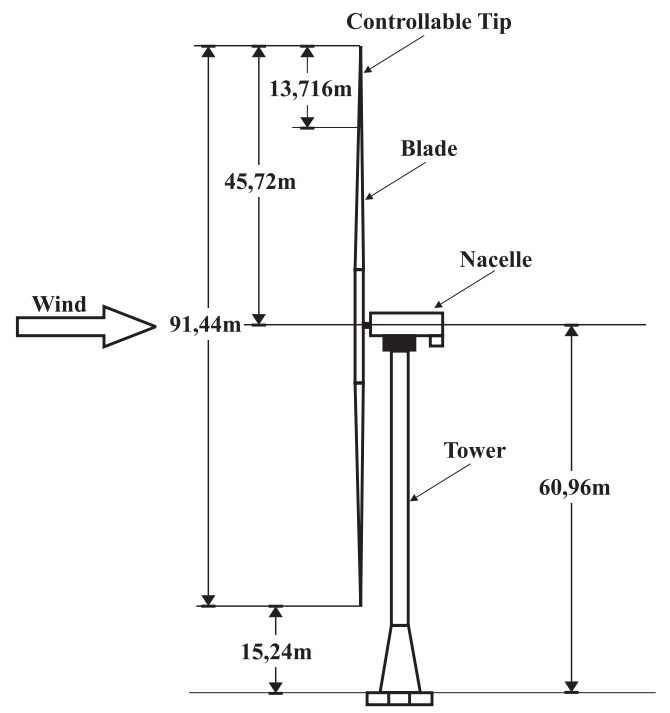

Fig. 8. Horizontal Axis WT used in computational simulations. each, which normalized data in relation to its rated values (2.5 MW and $17 \mathrm{RPM}$ ) are presented in Table 1. The WECS is simulated using the nonlinear model presented in section 2 . The control structure is designed using the nominal linearized model described in section 3, considering a sample interval of $T_{S}=5 \mathrm{~ms}$ and a nominal wind speed of $\bar{V}=6.7 \mathrm{~m} / \mathrm{s}$. The weights for LQR are chosen as $w_{1}=500, w_{2}=500, w_{3}=100, w_{4}=100, w_{5}=100$ and $w_{6}=1$. The covariance matrices for the Kalman filter design are selected as $\mathbf{V}=\varepsilon \psi^{\prime} \psi$ and $\mathbf{W}=10^{-3}$. Using the LTR procedure, the parameter $\varepsilon$ is adjusted to $10^{5}$ to obtain a good properties recovery. The Kalman predictor is designed selecting the covariance matrices as $\mathbf{V}_{\text {pred }}=T_{s}^{2} \psi \psi^{\prime}$ and $\mathbf{W}_{\text {pred }}=\alpha^{2}$. The frequency response of the open-loop control system can be verified by Bode diagrams shown in Fig. 9, where the infinite gain and phase margins indicate a stable close-loop control system.

The first simulated event is the wind gust shown in Fig. 10 . Its is observed in Fig. 11 that the LQG/LTR controller provides an accurate adjustment for generator speed $\omega_{g}$. Thus, the WECS operates practically closed at constant $\lambda$ as shown in Fig. 12. Since system losses are not considered, the speed reference obtained by the Kalman predictor is slightly below the ideal reference. None torsional mode is excited by this wind gust. The control system imposes an adequate attenuation for wind flutuaction and ripple torque, avoiding the propagation of dynamical loads to other parts of the WECS, as can be observed in Figs. 11 and 13.

Since a WT can be submitted to sudden wind variations, the second simulated event to verify the closed-loop dynamics is a wind step. Considering the operation at a mean wind speed of $6.7 \mathrm{~m} / \mathrm{s}$, it is suddenly varied to $8.9 \mathrm{~m} / \mathrm{s}$ after $20 \mathrm{~s}$ as shown the Fig. 14. In face of the increase on wind speed, the controller increases the generator speed (Fig. 15) to maintain $\lambda$ constant (Fig. 16), assuring the WECS operation almost in maximum efficiency on energy conversion. None torsional mode is excited by this wind step. It is observed in Figs. 15 and 17 that the control system continues imposing an adequate attenuation for wind flutuaction and ripple torque for a wind speed of $8.9 \mathrm{~m} / \mathrm{s}$. In this context, the fatigue on drive-train components is reduced, improving the quality of generated energy.

Table 1

WT normalized data.

\begin{tabular}{ll}
\hline$J_{t}=37.413$ & $D_{t}=2.024 \times 10^{-2}$ \\
$K_{h g}=28.4$ & $D_{h g}=1.831$ \\
$J_{g}=2.091$ & $D_{g}=3.01 \times 10^{-2}$ \\
\hline
\end{tabular}


Bode Diagram

$\mathrm{Gm}=\operatorname{lnf} \mathrm{dB}($ at $\operatorname{lnf} \mathrm{rad} / \mathrm{sec}), \mathrm{Pm}=\operatorname{lnf}$
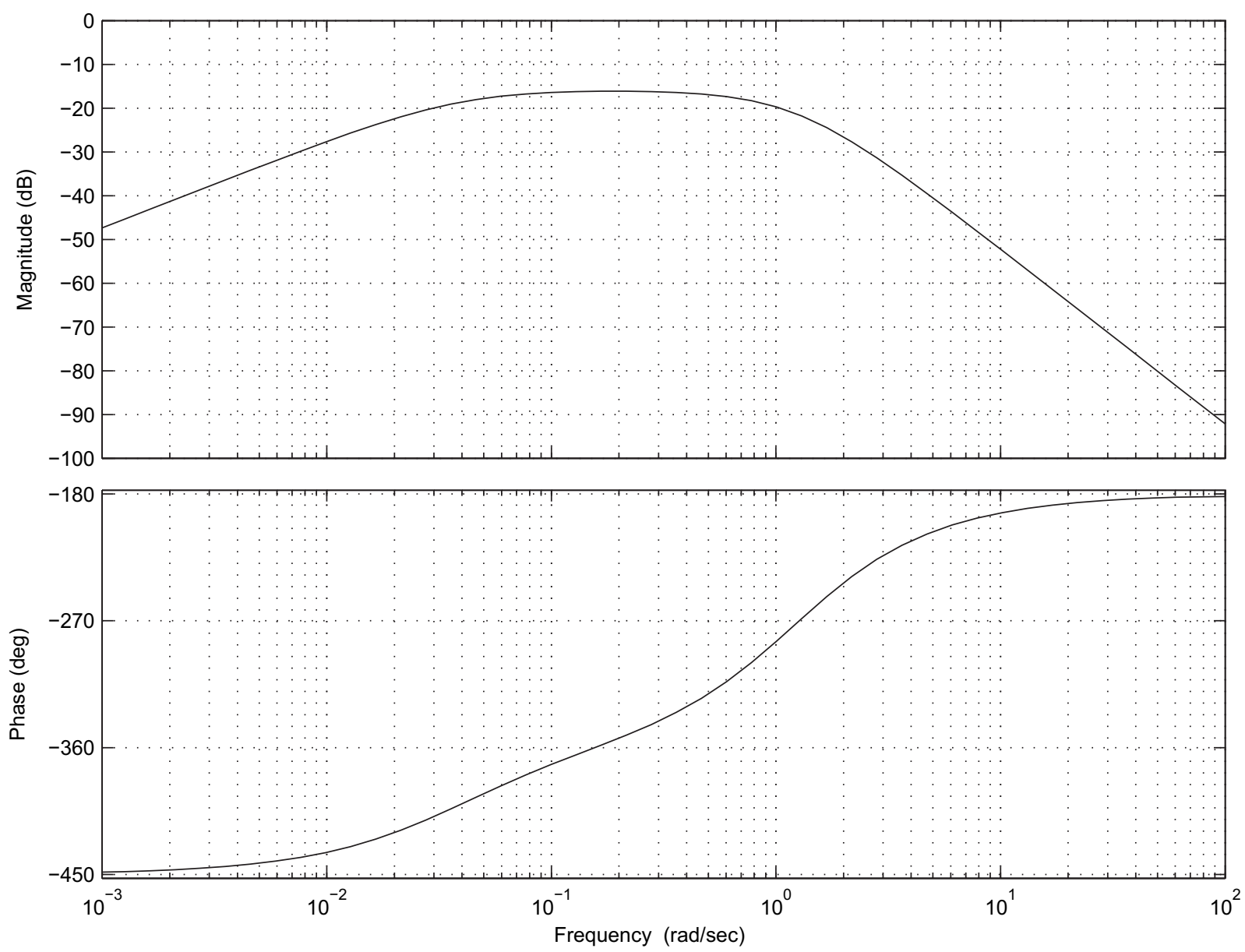

Fig. 9. Bode diagrams for open-loop WECS control system.

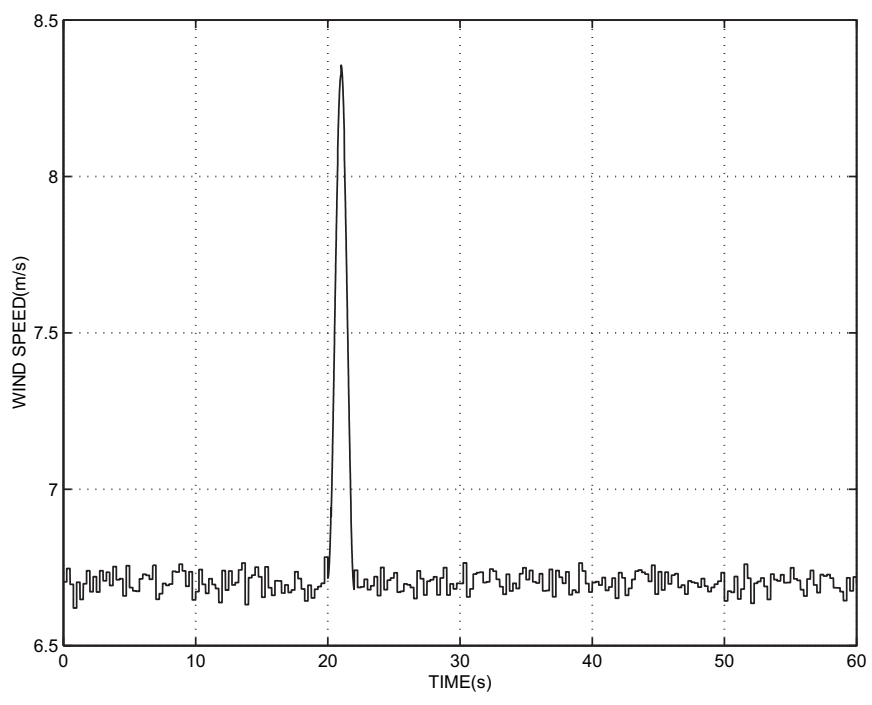

Fig. 10. Wind gust.

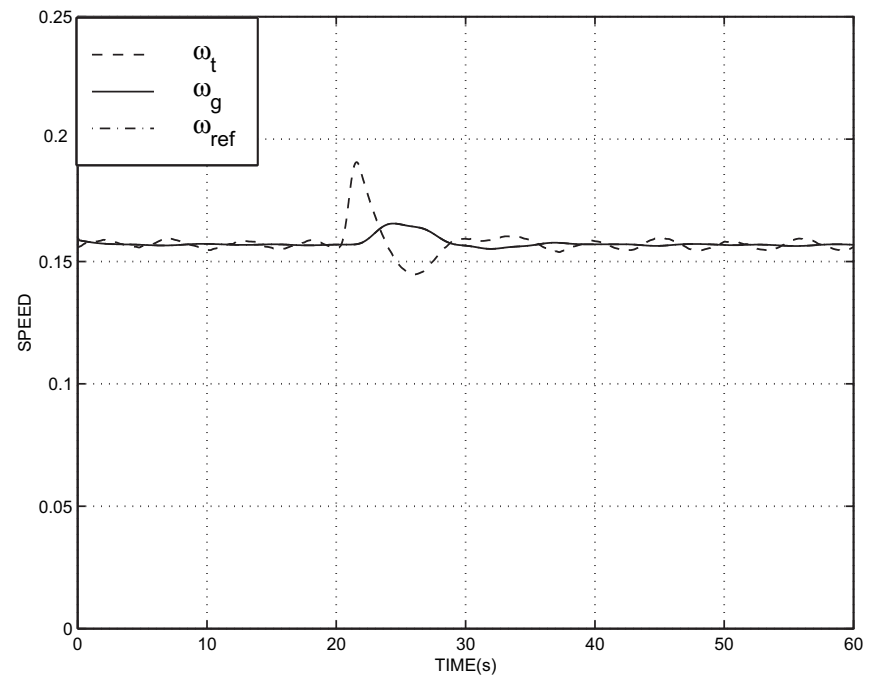

Fig. 11. Normalized speeds for the wind gust. 


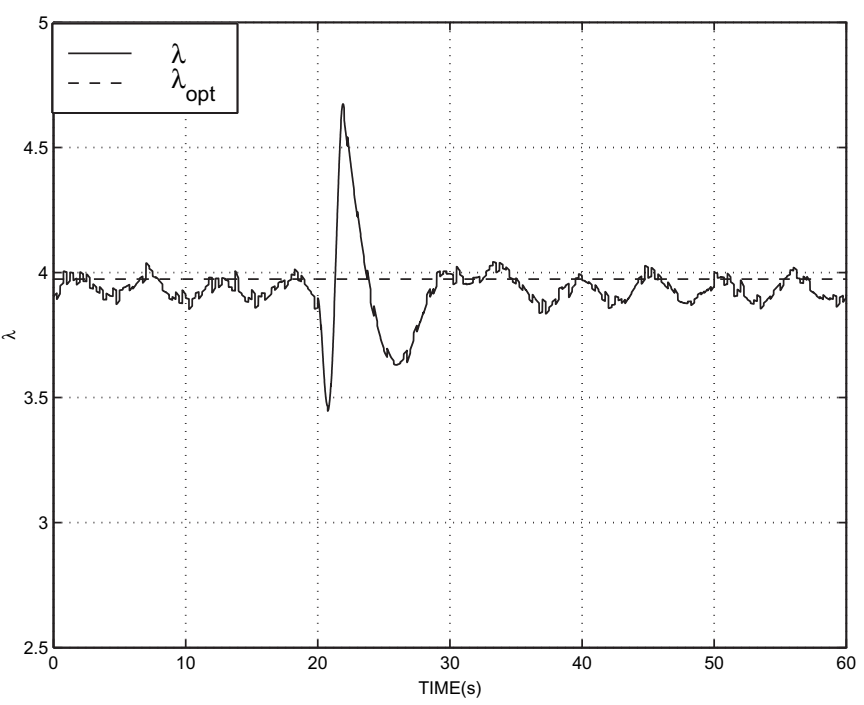

Fig. 12. Tip-speed ratio for the wind gust.

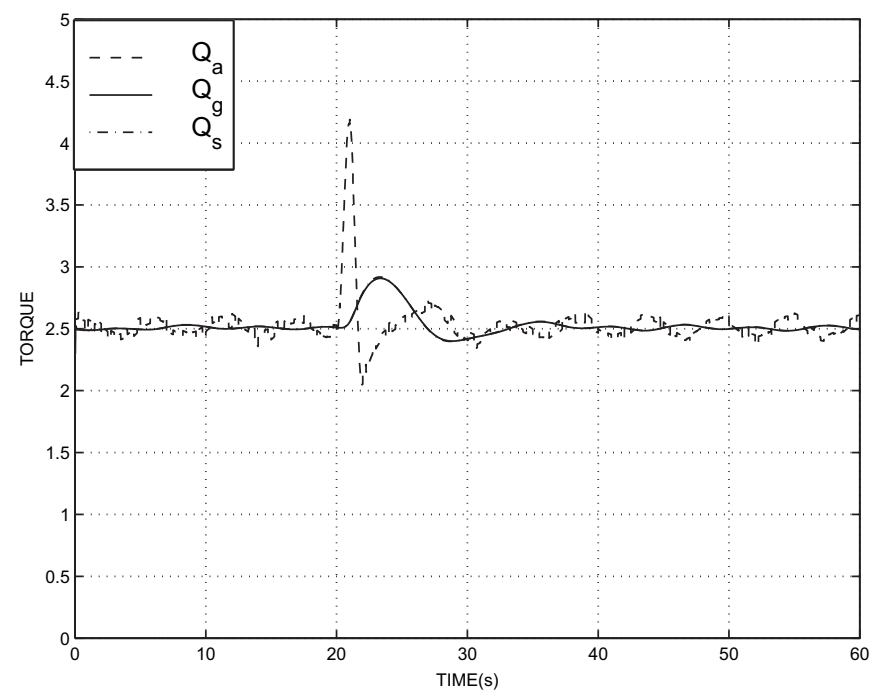

Fig. 13. Torques for the wind gust.

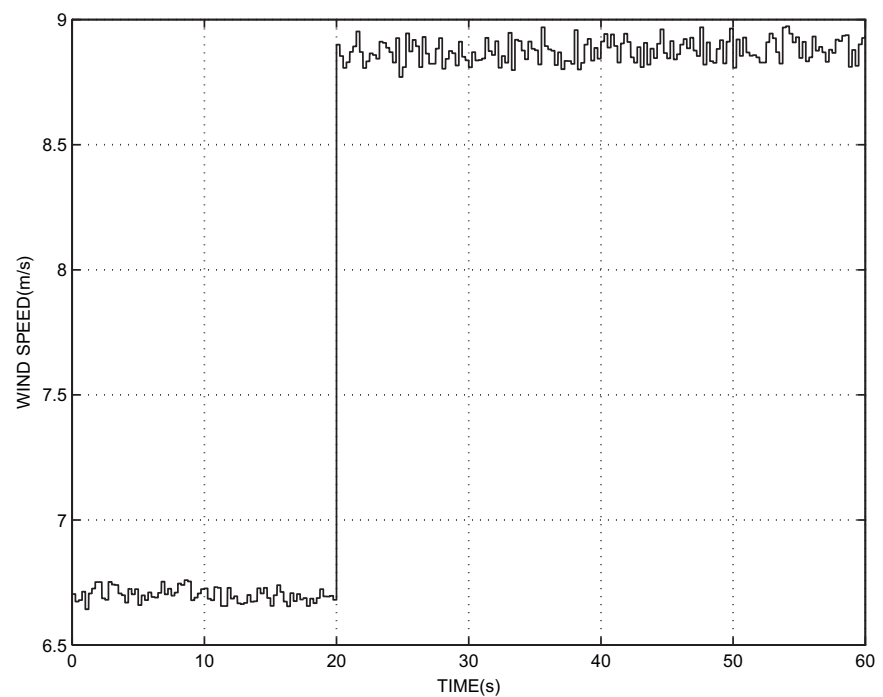

Fig. 14. Wind step.

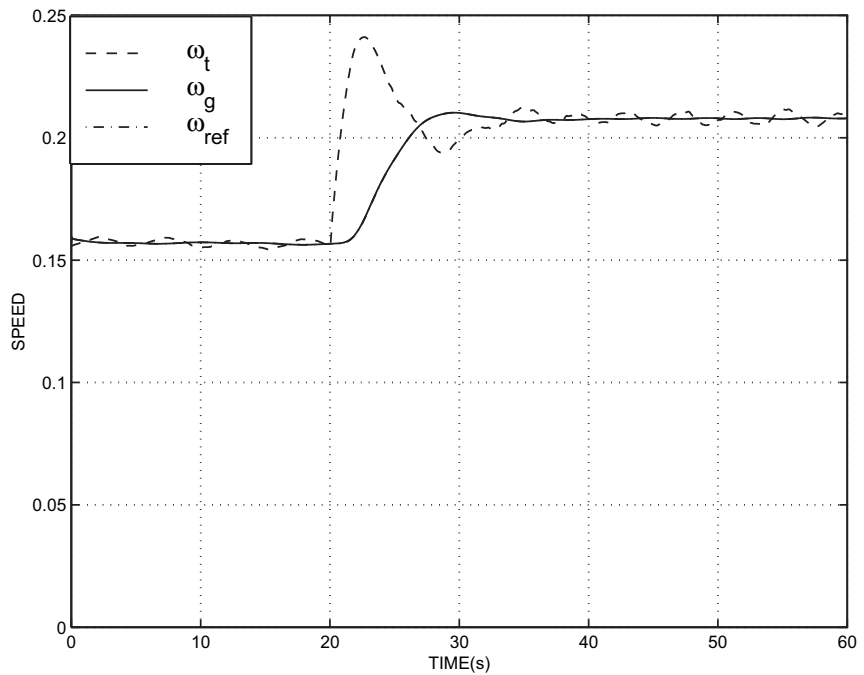

Fig. 15. Normalized speeds for a step on wind speed.

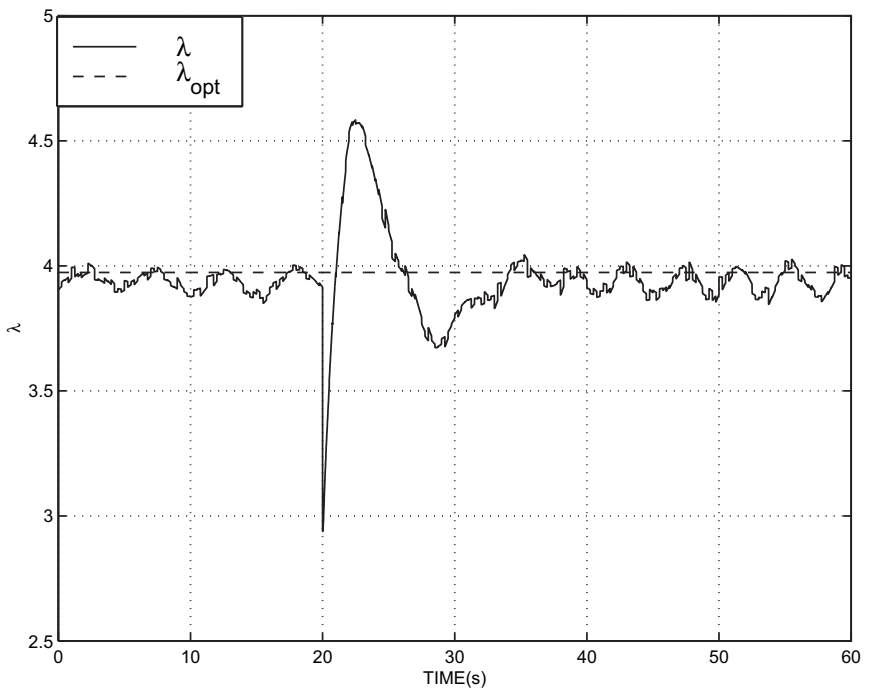

Fig. 16. Tip-speed ratio for a step on wind speed.

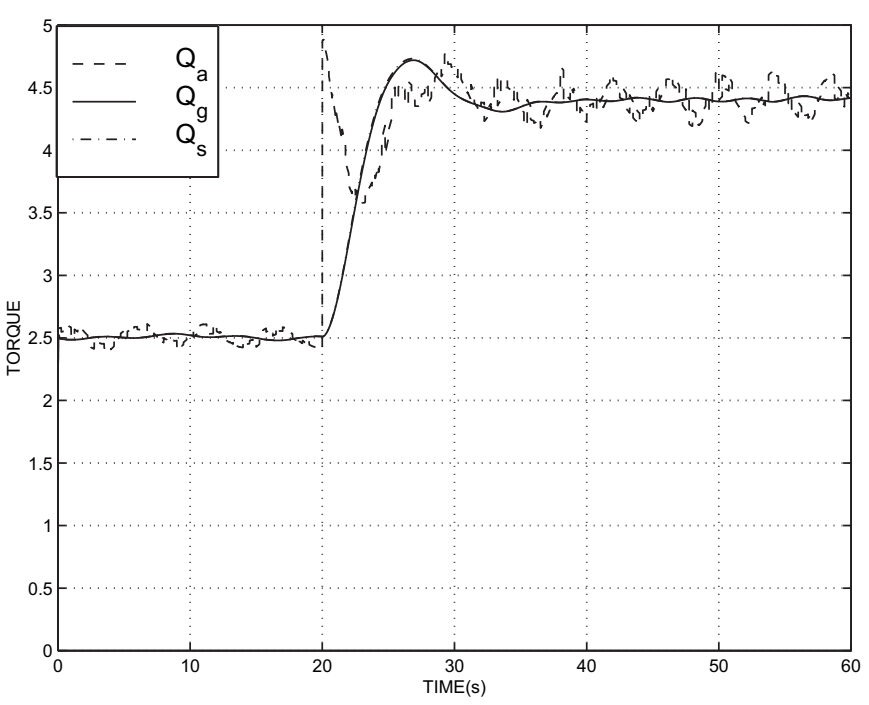

Fig. 17. Torques for a step on wind speed. 


\section{Conclusions}

This paper presents a sensorless control for a variable speed WT operating at partial load, that feeds an electrical load through static power converters. The speed reference is obtained from a Kalman predictor, which estimates the aerodynamic torque and avoids the direct wind speed measurement. This procedure is very attractive, since the complexity and the uncertainty of wind measurement can compromise the robustness of the control system. The LQG/LTR methodology was applied to design an optimal discrete-time feedback controller for a WECS. Since this methodology automatically assures the stability margin for the close-loop system, the control system is designed to obtain an effective performance for dynamical response through an adequate choice of targets and weighting matrices, establishing a formal cost function to be minimized by control action. The objective is to increase the efficiency on energy conversion, reducing the detrimental dynamic loads caused by wind fluctuation and ripple torque. Considering a consistent nonlinear dynamical model for WT system as the real plant, a wind gust and a step variation on wind speed are simulated to verify the closed-loop system performance. The simulation results have shown that the proposed sensorless control structure provides a WECS operation near the maximum efficiency point, reducing the influence of vibrations and ripple torque and, consequently, the stress over the mechanical drive system.

\section{Acknowledgments}

The authors gratefully acknowledge the financial support of the National Counsel of Technological and Scientific Development (CNPq), State of Minas Gerais Research Foundation (FAPEMIG), Gorceix Foundation and Federal University of Ouro Preto.

\section{References}

[1] Lefevbre S, Dubé B. Control system analysis and design for an aerogenerator with eigenvalue methods. IEEE Trans Power Syst 1988;3(4):1600-8.

[2] Dessaint L, Nakra H, Mukhedkar D. Propagation and elimination of torque ripple in a wind energy conversion system. IEEE Trans Energy Conversion 1986;1(2):104-12.

[3] Leithead WE, de la Salle S, Reardon D. Role and objectives of control for wind turbines. IEE Proc Pt C 1991;138(2):135-48.

[4] Østergaard K, Stoustrup J, Brath P. Linear parameter varying control of wind turbines covering both partial load and full load conditions. Int J Robust Nonlinear Control 2009;19:92-116.

[5] Geng H, Yang G. Robust pitch controller for output power levelling of variablespeed variable-pitch wind turbine generator systems. IET Renewable Power Generation 2009;3(2):168-79.

[6] Grimble MJ. Robust industrial control - optimal design approach for polynomial systems, systems and control engineering. New York: Prentice Hall International; 1994.

[7] Maciejowski JM. Multivariable feedback design. Wokingham, England: Addison-Wesley Publishing Company Inc.; 1989.

[8] Novak P, Ekelund T, Jovik I, Schmidtbauer B. Modeling and control of variablespeed wind-turbine drive-system dynamics. IEEE Control Syst 1995;15 (4):28-38.
[9] Leithead WE. Variable speed operation - does it have any advantages? Wind Eng 1989;13(6):302-14

[10] Rocha R, Resende P, Silvino J.L, Bortolus M.V. Control for a wind turbine without wind measurement. In: Proceedings XVI COBEM, ABCM, Uberlândia, Brazil; 2001.

[11] Wasynczuk O, Man DT, Sullivan JP. Dynamic behaviour of a class of wind turbine generators during random wind fluctuations. IEEE Trans. Power Apparatus Syst 1981;100(6):2837-45.

[12] Leith DJ, Leithead WE. Implementation of wind turbine controllers. Int J Control 1997;66(3):349-80.

[13] Rohatgi J, Pereira A. Modeling wind turbulence for the design of large wind turbines - a preliminary analysis. In: IV CEM-NNE; 1996. p. 735-9.

[14] Anderson PM, Bose A. Stability simulation of wind turbine systems. IEEE Trans Power Apparatus Syst 1983;102(12):3791-5.

[15] Freris LL, editor. Wind energy conversion systems. United King: Prentice Hall Inc.; 1990.

[16] Golding EW. The generation of electricity by wind power. London, UK: E. \& F.N. Spon LTD; 1977.

[17] Raina G, Malik OP. Variable speed wind energy conversion using synchronous machine. IEEE Trans Aerospace Electronic Syst 1985;21(1):100-4.

[18] Hwang HH, Gilbert LJ. Synchronization of wind turbine generators against an infinite bus under gusting wind conditions. IEEE Trans Power Apparatus Syst 1978;97(2):536-44.

[19] Medeiros A, Simões FJ, Lima AMN, Jacobina CB. Modelagem aerodinâmica de turbinas eólicas de passo variável. Florianópolis, Brazil: VI ENCIT/VI LATCYM, ABCM; 1996. pp. 25-30.

[20] Hori Y, Sawada H, Chun Y. Slow resonance ratio control for vibration suppression and disturbance rejection in torsional system. IEEE Trans Ind Electronics 1999;46(1):162-8.

[21] Lefebvre S, Dessaint L, Dubé B, Nakra H, Pérocheau A. Simulator study of a vertical axis wind turbine generator connected to a small hydro network. IEEE Trans Power Apparatus Syst 1985;104(5):1095-101.

[22] Rocha R, Resende P, Silvino J.L, Bortolus M.V. Control of stall regulated wind turbine through $\mathrm{H} \infty$ loop shaping method. In: 2001 IEEE conference on control applications CCA2001, vol. 1. Mexico City; 2001. p. 925-9.

[23] Rocha R, Martins Filho L.S. A multivariable $\mathrm{H} \infty$ control for wind energy conversion system. In: 2003 IEEE conference on control applications CCA2003, vol. 1. Istambul; 2003. p. 206-11.

[24] Hinrinchsen E, Nolan P. Dynamics and stability of wind turbine generators. IEEE Trans Power Apparatus Syst 1982;101(8):2640-8.

[25] Bratcu A, Munteanu I, Ceangã E. Optimal control of wind energy conversion systems: from energy optimization to multi-purpose criteria - a short survey. In: 16th Mediterranean conference on control and automation. Ajaccio, France; 2008. p. 759-66.

[26] Anderson BDO, Moore JB. Optimal control - linear quadratic methods. New Jersey, USA: Prentice Hall Inc.; 1990.

[27] Maciejowski JM. Asymptotic recovery for discrete-time systems. IEEE Trans Automatic Control 1985;30(6):602-5.

[28] Bose B. Power electronics and variable frequency drives: technology and applications. IEEE Press; 1997.

[29] Lefley P.W, Peasgood W, Ong R, Wong J.K.J. Sensorless close loop control of a slip ring induction machine using adaptive signal processing. In: 14th annual applied power electronics conference and exposition, APEC99; 1999.

[30] Cárdenas R, Peña R, Asher G, Cilia J. Sensorless control of induction machines for wind energy applications. In: 33rd annual power electronics specialists conference, PESC2002; 2002.

[31] Cárdenas R, Peña R, Asher G, Clare J. Power smoothing in wind generation systems using a sensorless vector controlled induction machine driving a flywheel. IEEE Trans on Energy Conversion;19(1).

[32] Hilairet M, Auger F. Frequency estimation for sensorless control of induction motors. In: Proceedings of 2001 IEEE conference on acoustic, speech and signal processing; 2001.

[33] Holtz J. Sensorless position control of induction motors - an emerging technology. IEEE Trans on Industrial Electronics;45(6).

[34] Phipps W, Al-Bahadly I. Sensorless speed control in induction motor drives. In: Proceedings of 1st IEEE international workshop on electronic design, test and applications; 2002. 